\title{
ON THE DETERMINATION OF A FUNCTION IN THE PLANE BY ITS INTEGRALS OVER STRAIGHT LINES ${ }^{1}$
}

\author{
JOHN W. GREEN
}

In the December 1957 issue of the American Mathematical Monthly (page 750) there appears the solution by D. J. Newman of the following problem proposed by him: Let $f(x, y)$ be coniinuous and summable over the plane, and let the line integral $\int f d s$ be zero when extended over any infinite straight line; prove that $f \equiv 0$.

It was pointed out in the Monthly that this problem, which is related to the projections of probability distributions, had been stated and solved by A. Rényi [1]. In this note I shall give another short solution of this problem based on the use of the Newtonian potential, and show how the method applies to the solution of another problem raised by Renyi in the same paper.

Let $U(x, y, z)$ be the Newtonian potential of the distribution of charge in the $x-y$ plane with charge density $f(x, y)$, of which we momentarily assume $|f| \leqq K$. Let polar coordinates $(r, \theta)$ be introduced in the $x-y$ plane at an arbitrary pole $\left(x_{0}, y_{0}\right)$, and set $f(x, y)$ $=g(r, \theta)$. Then

$$
U\left(x_{0}, y_{0}, 0\right)=\iint \frac{f(x, y) d x d y}{r}=\int_{0}^{\pi} d \theta \int_{-\infty}^{\infty} g(r, \theta) d r=0,
$$

since the inner integral is just the line integral of $f(x, y)$ over the line through $\left(x_{0}, y_{0}\right)$ corresponding to slope angle $\theta$. That is, $U$ vanishes identically in the $x-y$ plane. It is harmonic outside this plane and clearly bounded in all space by the potential at its center of a uniform circular disk of density $K$ and total mass equal to $\iint|f(x, y)| d x d y$. Analytic continuation by reflection of $U$ across the $x-y$ plane yields a function harmonic and bounded in all space and vanishing on the $x-y$ plane. Thus $U \equiv 0$, as follows easily from Harnack's principle, and since $f$ is equal to $(4 \pi)^{-1}$ times the discontinuity of $U_{z}$ across the plane, $f \equiv 0$.

The requirements that $f$ be continuous and bounded are not essential. If $f$ is merely summable, let $f_{r}(x, y)$ be the circular area mean of

Received by the editors February 22, 1958.

1 The preparation of this paper was sponsored in part by the Office of Naval Research. Reproduction as a whole or in part is permitted for the purposes of the United States Government. 
$f$ over a circle with center $(x, y)$ and radius $r$. Then $f_{r}$ is continuous and bounded, and also has zero integral along straight lines, as follows from a permissible interchange of order of integration. Thus $f_{r} \equiv 0$, and since $f_{r} \rightarrow f$ almost everywhere when $r \rightarrow 0, f=0$ almost everywhere.

The problem raised by Rényi is based on the observation that if $f(x, y)=1 /\left(1-x^{2}-y^{2}\right)^{1 / 2}$ for $x^{2}+y^{2}<1$, then $\int f(x, y) d s=\pi$ when the integral is extended over any chord of the unit circle. The problem is the following: Does there exist a convex curve $C$ other than the circle, and an integrable function $f$ defined interior to $C$ and not identically zero, such that $\int f d s=$ constant over all chords of $C$ ?

It may be interesting to note that this problem, which also occurred to Hans Lewy and the author and was solved by them in 1945 , comes up in a completely different context from probability distributions. The so-called "plank problem" of Tarski is to show that if a convex set in the plane is covered by $n$ planks (a plank is an infinite strip between two parallel lines) of width $w_{i}$, then $\sum_{i}^{n} w_{i}$ is at least the width of the narrowest single plank covering the set. This famous problem, which has subsequently been solved by T. Bang [2], had not been solved in 1945; however there has long been an extremely simple and ingenious proof in the case the convex set is a circle. This proof depends on constructing a spherical surface of which the given circle is an equator, and noting that the vertical projection of a plank gives on the sphere a zone, whose area depends only on its width, but not on its position. This latter fact depends on the property of $1 /\left(1-x^{2}-y^{2}\right)^{1 / 2}$ mentioned above. Conversely, if corresponding to a given $C$ one could construct a function $f$ as described in Rényi's problem, he would have a solution of the plank problem for that $C$.

The solution of Rényi's problem turns out in the negative.

TheOREM. Let $C$ be a convex curve of width 2 and $f$ a summable function over its interior, with $\int f d s=k$ over all chords of $C$. Then either $f=0$ almost everywhere or else $C$ is a circle, and $f \equiv k / \pi\left(1-x^{2}-y^{2}\right)^{1 / 2}$.

If $k=0$, define $f=0$ outside and on $C$. The solution to the Monthly problem applies and $f=0$ almost everywhere. Now assume $k \neq 0$; it may as well be taken to be 1 .

Let $U(x, y, z)$ be the Newtonian potential of the planar distribution inside $C$ with density $f$. Let $(x, y, 0)$ be a point in the $x-y$ plane outside $C$, and $(r, \theta)$ polar coordinates with pole at that point. If $f=g(r, \theta)$,

$$
U(x, y, 0)=\int d \theta \int g(r, \theta) d r
$$


where the double integral extends over the interior of $C$. The inner integral equals 1 in the $\theta$-interval corresponding to those rays from $(x, y, 0)$ which intersects the interior of $C$ and equals 0 outside that interval. Thus the double integral equals $\phi(x, y)$, the angle subtended by $C$ at $(x, y, 0) ; \phi(x, y)=U(x, y, 0)$. Inside $C, U=\pi$; thus $U$ is an equilibrium potential for the lamina bounded by $C$.

Let the harmonic potential function $U(x, y, z)$ be expanded in a series of spherical harmonics, uniformly convergent near infinity. If $z$ is set equal to zero, the expansion is of the form

$$
U(x, y, 0)=\phi(x, y)=\frac{a_{0}}{\left(x^{2}+y^{2}\right)^{1 / 2}}+\frac{a_{1} x+b_{1} y}{\left(x^{2}+y^{2}\right)^{3 / 2}}+\cdots .
$$

Let $p(\theta)$ be the support function of $C$; that is, $p(\theta)=\sup (x \cos \theta$ $+y \sin \theta)$ for all $(x, y)$ interior to $C$. Let $\theta_{1}$ and $\theta_{2}$ define the directions of the rays normal to the two supporting lines to $C$ drawn from $(x, y)$. These supporting lines have equations

$$
\begin{aligned}
& x \cos \theta_{1}+y \sin \theta_{1}=p\left(\theta_{1}\right), \\
& x \cos \theta_{2}+y \sin \theta_{2}=p\left(\theta_{2}\right),
\end{aligned}
$$

and for proper determination of $\theta_{1}$ and $\theta_{2}$ (see Fig. 1),

$$
\phi=\theta_{2}-\theta_{1}-\pi \text {. }
$$

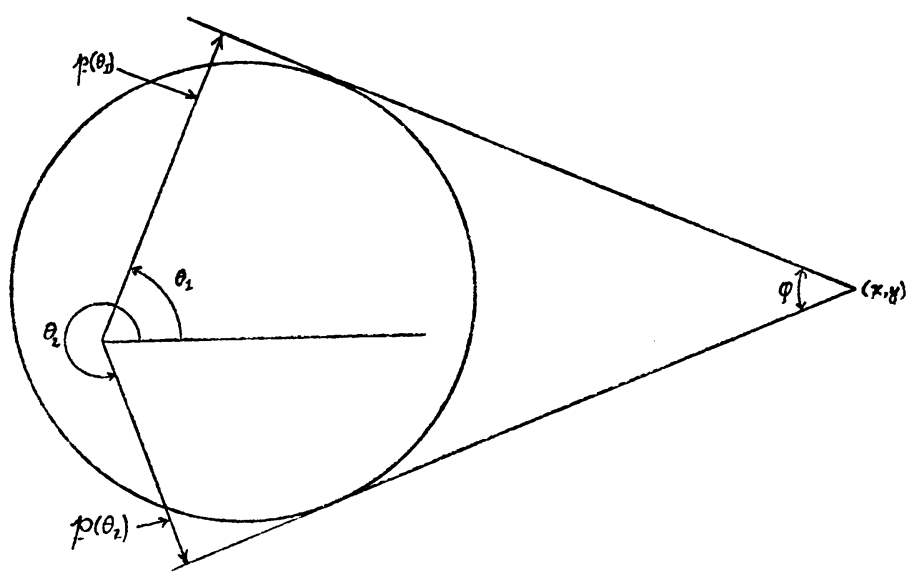

FIG. 1

If equations (2) are solved for $x$ and $y$, we obtain, making use of (3) and writing $p\left(\theta_{i}\right)=p_{i}$,

$$
x=\frac{p_{2} \sin \theta_{1}-p_{1} \sin \theta_{2}}{\sin \phi}, \quad y=\frac{p_{1} \cos \theta_{2}-p_{2} \cos \theta_{1}}{\sin \phi}
$$


and

$$
x^{2}+y^{2}=\frac{p_{1}^{2}+p_{2}^{2}+2 p_{1} p_{2} \cos \phi}{\sin ^{2} \phi} .
$$

Now let $x^{2}+y^{2} \rightarrow \infty$, keeping $\theta_{1}$ constant. Clearly $\phi \rightarrow 0$ and $\theta_{2} \rightarrow \theta_{1}+\pi$. From (1),

$$
\begin{aligned}
a_{0}=\lim \phi \cdot\left(x^{2}+y^{2}\right)^{1 / 2} & =\lim \frac{\phi \cdot\left(p_{1}^{2}+p_{2}^{2}+2 p_{1} p_{2} \cos \phi\right)^{1 / 2}}{\sin \phi} \\
& =p\left(\theta_{1}\right)+p\left(\theta_{1}+\pi\right) .
\end{aligned}
$$

Since $\theta_{1}$ is arbitrary, it follows that

$$
p(\theta)+p(\theta+\pi)=a_{0},
$$

or $C$ is of constant width.

Also it is clear that as $x^{2}+y^{2} \rightarrow \infty$ with constant $\theta_{1}$,

$$
\begin{aligned}
& \frac{x}{\left(x^{2}+y^{2}\right)^{1 / 2}} \rightarrow \cos \left(\theta_{1}-\frac{\pi}{2}\right)=\sin \theta_{1}, \\
& \frac{y}{\left(x^{2}+y^{2}\right)^{1 / 2}} \rightarrow \sin \left(\theta_{1}-\frac{\pi}{2}\right)=-\cos \theta_{1} .
\end{aligned}
$$

Hence from (1),

$$
\left\{\phi-\frac{a_{0}}{\left(x^{2}+y^{2}\right)^{1 / 2}}\right\}\left\{x^{2}+y^{2}\right\} \rightarrow a_{1} \sin \theta_{1}-b_{1} \cos \theta_{1} .
$$

The function $p(\theta)$ has right and left hand derivatives everywhere [3]; hence using (3) and (5),

$$
\begin{aligned}
p_{2} & =p\left(\theta_{2}\right)=p\left(\theta_{1}+\phi+\pi\right)=a_{0}-p\left(\theta_{1}+\phi\right) \\
& =a_{0}-\left(p\left(\theta_{1}\right)+p^{\prime+}\left(\theta_{1}\right) \phi+o(\phi)\right) .
\end{aligned}
$$

If this expression for $p_{2}$ is substituted in (4), it is easily computed that

$$
\left\{\phi-\frac{a_{0}}{\left(x^{2}+y^{2}\right)^{1 / 2}}\right\}\left\{x^{2}+y^{2}\right\}=-a_{0} p^{\prime+}\left(\theta_{1}\right)+o(1),
$$

and so from (6),

$$
-a_{0} p^{\prime+}\left(\theta_{1}\right)=a_{1} \sin \theta_{1}-b_{1} \cos \theta_{1},
$$

for each $\theta_{1}$. Since $p^{\prime+}$ is now seen to be continuous, $p^{\prime}$ itself exists and

$$
-a_{0} p^{\prime}(\theta)=a_{1} \sin \theta-b_{1} \cos \theta .
$$

Thus $p$ is of the form 


$$
p=A \cos \theta+B \sin \theta+E,
$$

and $C$ is a circle, of which the equilibrium density is well known to be of the form given.

Added in proof. Since this paper was prepared, there has appeared another solution of the Monthly problem described in the opening paragraph, by A. S. Besicovitch: A uniqueness theorem and a problem in integration, J. London Math. Soc. vol. 33 (1958) pp. 82-84.

\section{REFERENCES}

1. A. Rényi. On projections of probability distributions. Acta Math. Sci. Hungar. vol. 3 (1952) pp. 131-142.

2. T. Bang. A solution of the "plank problem," Proc. Amer. Math. Soc. vol. 2 (1951) pp. 990-992.

3. John W. Green. Support, convergence, and differentiability properties of generalized convex functions, Proc. Amer. Math. Soc. vol. 4 (1953) pp. 391-396.

University of California at los Angeles 